\title{
Longitudinally vibrating elastic rods with locally and non-locally reacting viscous dampers
}

\author{
Şefaatdin Yüksel ${ }^{\mathrm{a}, *}$ and Uğur Dalli ${ }^{\mathrm{b}}$ \\ ${ }^{a}$ Department of Mechanical Engineering, Faculty of Engineering and Architecture, Gazi University, 06570 \\ Maltepe, Ankara, Turkey \\ ${ }^{\mathrm{b}}$ ASELSAN Inc., MGEO Division, 06511 Akyurt, Ankara, Turkey
}

Received 10 February 2004

Revised 18 June 2004

\begin{abstract}
Eigencharacteristics of a longitudinally vibrating elastic rod with locally and non-locally reacting damping are analyzed. The rod is considered as a continuous system and complex eigenfrequencies are determined as solution of a characteristic equation. The variation of the damping ratios with respect to damper locations and damping coefficients for the first four eigenfrequencies are obtained. It is shown that at any mode of locally or non-locally damped elastic rod, the variation of damping ratio with damper location is linearly proportional to absolute value of the mode shape of undamped system. It is seen that the increasing damping coefficient does not always increase the damping ratio and there are optimal values for the damping ratio. Optimal values for external damping coefficients of viscous dampers and locations of the dampers are presented.
\end{abstract}

\section{Introduction}

Damping force characterization, force transmissibility reduction and displacement minimization in vibrating structures are active research areas in structural dynamics. Dynamic response of vibrating structures frequently modeled as simply supported beams such as bridges and long span roofs can be reduced considerably by use of appropriate viscous dampers. Uses of viscous dampers in elastic structures such as flexible beams, long steel cables used in cable-stayed bridges and in other structures have proven to be of significant interest in many applications and several studies have investigated the use of optimal viscous dampers to eliminate the axial vibrations of structures.

Zarek and Gibbs [1] obtained the complex eigenvalues and mode shapes of a damped beam with generic end conditions. A numerical procedure to evaluate the complex frequencies and mode shapes of a simply supported beam with two rotational viscous dampers at its ends was presented by Oliveto et al. [2]. They stated that an appropriate choice of the damper constant allows for the maximum reduction of the dynamic response or for an optimal overall design. Yang and $\mathrm{Wu}$ [3] proposed an exact closed form solution method for transient analysis of general one-dimensional distributed systems subject to arbitrary external, initial and boundary disturbances. The proposed method was illustrated on a cantilever beam with end mass, viscous damper and spring.

Cables supported by viscous dampers find application areas in the design of bridges and similar structures. Kovacs [4] studied a taut cable supported with a viscous damper and used a semi-empirical interpolation to obtain maximum modal damping ratio. Pacheco et al. [5] proposed a universal curve relating the modal damping ratio, the

\footnotetext{
* Corresponding author: Dr. Şefaatdin Yüksel, Department of Mechanical Engineering, Faculty of Engineering and Architecture, Gazi University, 06570 Maltepe, Ankara, Turkey. Fax.: +90 312232 0427; E-mail: syuksel@gazi.edu.tr.
} 
mode number, size and location of the damper, and the cable parameters consisting of span, mass and fundamental frequency. They obtained the curve from a numerical complex eigenvalue analysis and illustrated its use for some example situations to find the value of optimal external damping constant. Krenk [6] analyzed the damping of a vibrating string by an isolated viscous damper in terms of complex modes. The asymptotic solution proposed for the damping ratio of the lower modes is valid for damper location close to one support. In addition to these works related to the cable, Casarella and Laura [7] formulated an analytical expression for the viscous drag on a cable oscillating with longitudinal and torsional motion and presented an approximate value for the drag coefficient based on a linear damping law. Goeller and Laura [8] analyzed the dynamic stresses and displacements in a cable having external damping due to fluid action and internal damping due to viscoelastic material properties. The cable is considered to be made up of two segments of different materials and subjected to longitudinal excitation simulating ocean-wave motion.

The studies on the longitudinal vibrations of viscously damped bars show similarity to those of the cable. A closed form eigensolution for a longitudinally vibrating bar fixed at one end and constrained by a lumped viscous damper at the other was developed by Singh et al. [9]. Hull [10] analyzed the same problem with an external axial force and developed a closed form series solution for the axial wave equation of this longitudinally vibrating bar with the same boundary conditions. Furthermore, Hizal and Gürgöze [11] presented a study dealing with a longitudinally vibrating rod fixed at one end and free at the other and damped viscously by a single damper in span. They studied the eigencharacteristics of the rod and compared the results by regarding the same system first as a continuous system and then as a discrete system. In a recent paper, Adhikari and Woodhouse [12] proposed methods to identify viscous and non-viscous damping models for vibrating systems using measured complex frequencies and mode shapes. In a recent study, Yüksel and Gürgöze [13] investigated the eigencharacteristics of continuous model of a longitudinally vibrating rod which is damped viscously by a single damper in span and fixed at both ends. An asymptotic solution was derived to obtain a simple explicit formula for calculating the complex eigenfrequencies and the damping ratio of the continuous model.

This paper considers the identification of the eigencharacteristics of a viscously damped longitudinally vibrating rod fixed at both ends. Although the finite element methods are capable of modeling complicated distributed systems, only a specific numerical result for a specific system parameter is obtained and a general closed-form solution, which would permit one to examine system response to changes in various parameters, is not produced with these methods. The problem can also be solved using dynamic stiffness method with nodal damping [14]. The purpose in this study is to develop alternative analytical solutions for the optimal values of the damping constant, the locations of the dampers and rod parameters in order to reduce the dynamic response of the system. Closed form solutions, if obtainable, are always desirable because of their efficiency, and physical insight in to the problem.

The eigencharacteristics of the rod is analyzed for the two alternative damping conditions. In the first, two arbitrary points of the rod are grounded through two separate viscous dampers. This is a common way of introducing damping to civil structures such as bridges and long span roofs. In the second, two arbitrary points of the rod are connected through a single viscous damper. This approach may better reduce the dynamic response of the system and may provide flexibility both in the design and in the installation of viscous dampers. Thus, the optimal values of viscous damping and damper locations may be best determined.

At first, a continuous model of the rod is considered and the complex eigenfrequencies are presented as the solution of a characteristic equation for the two alternatives of viscous damper conditions. Following that, the optimal values for external damping constant of viscous damper and the locations of the dampers are determined in order to reduce the dynamic response of the system. The results for both cases are compared and some numerical examples are given to illustrate the solutions. Finally, some implications of these results for damping identification are summarized.

\section{Mathematical model}

The mechanical systems to be considered are shown in Fig. 1. In case 1, a longitudinal vibrating elastic rod fixed at both ends is connected to the ground from certain points by two separate viscous dampers as shown in Fig. 1(a). Such a damping system is described as "locally reacting" since the damping force depends only on the absolute motion of the individual points [12]. In case 2, however, a single viscous damper connects two distinct points of the 

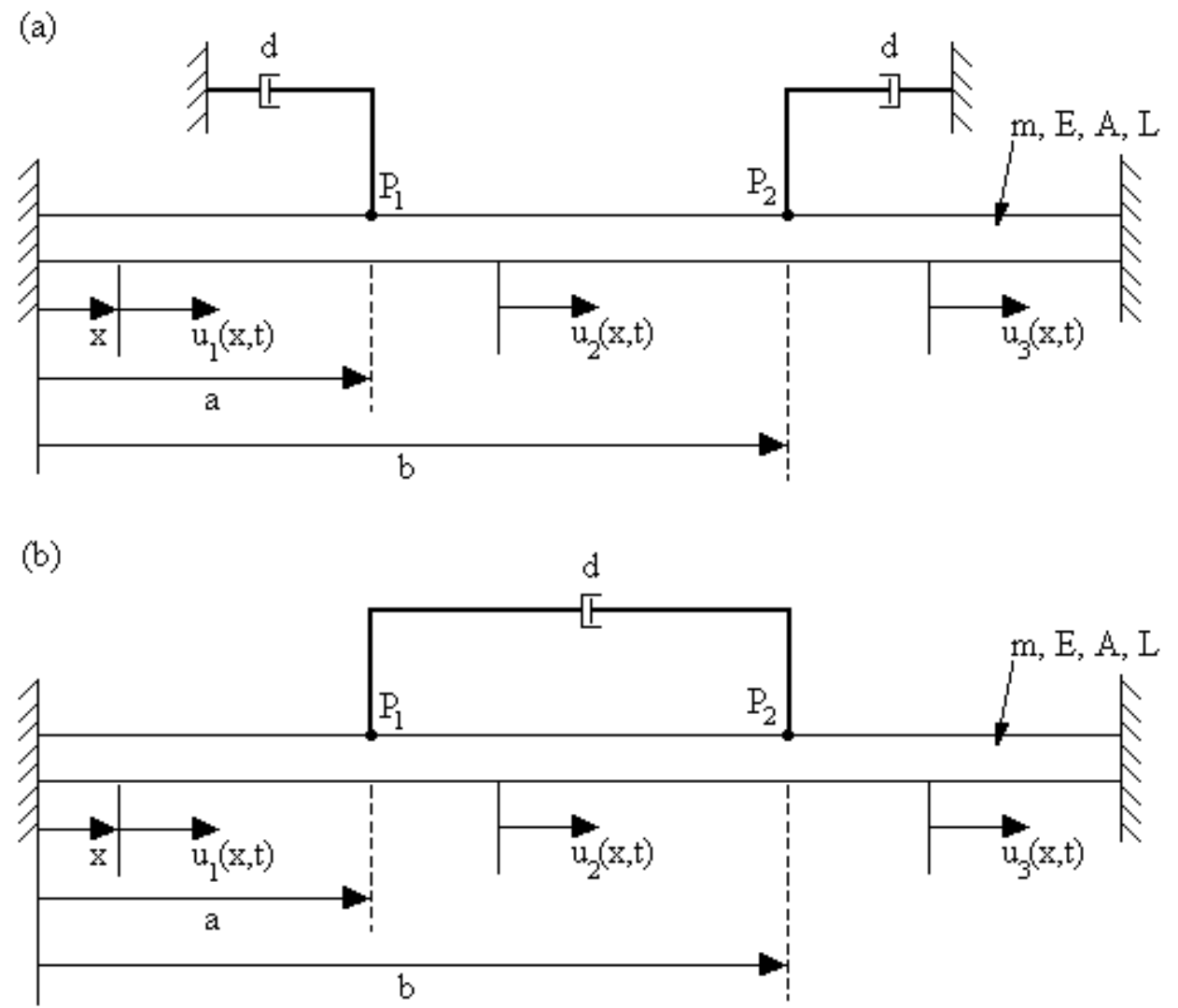

Fig. 1. Longitudinally vibrating elastic rods with (a) locally reacting damping and (b) non-locally reacting damping.

elastic rod as shown in Fig. 1(b). In this case, the damping force depends on the relative motion of the two points, and damping system is called as "non-locally reacting".

The following partial differential equation describes the longitudinal vibration of a uniform rod with no external force [15]:

$$
E A^{\partial^{2} u(x, t)} \begin{gathered}
\partial x^{2} \\
\partial \partial^{2} u(x, t) \\
\partial t^{2}
\end{gathered}
$$

where $u(x, t), m, E$ and $A$ denotes the axial displacement at a position $x$, mass per unit length, modulus of elasticity and cross sectional area of the rod, respectively. For this analysis, it is assumed that the rod is linearly elastic and has negligible internal structural damping.

For both non-locally and locally reacting damping systems, the rod is considered in three parts as shown in Fig. 1 . The axial displacements of these three sections, i.e., the left, middle and right sections are respectively represented by $u_{1}(x, t), u_{2}(x, t)$ and $u_{3}(x, t)$. The solution of the problem must satisfy the equation of motion, Eq. (1), over the whole length of the rod, as well as the following boundary and matching conditions:

$$
\begin{aligned}
& u_{1}(0, t)=0 \\
& u_{3}(L, t)=0 \\
& u_{1}(a, t)=u_{2}(a, t) \\
& u_{2}(b, t)=u_{3}(b, t)
\end{aligned}
$$


where $a$ and $b$ are the locations of the attachment points of the dampers at the left and right, respectively. In addition, the solution must satisfy the following matching conditions, for locally reacting damping system (Case 1),

$$
\begin{aligned}
& u_{1}^{\prime}(a, t)-u_{2}^{\prime}(a, t)+\left(\begin{array}{c}
d \\
E A
\end{array}\right) \dot{u}_{1}(a, t)=0 \\
& u_{2}^{\prime}(b, t)-u_{3}^{\prime}(b, t)+\left(\begin{array}{c}
d \\
E A
\end{array}\right) \dot{u}_{3}(b, t)=0
\end{aligned}
$$

and for non-locally reacting damping system (Case 2),

$$
\begin{aligned}
& u_{1}^{\prime}(a, t)-u_{2}^{\prime}(a, t)+\underset{E A}{d}\left[\dot{u}_{1}(a, t)-\dot{u}_{2}(b, t)\right]=0 \\
& u_{2}^{\prime}(b, t)-u_{3}^{\prime}(b, t)+{ }_{E A}^{d}\left[\dot{u}_{3}(b, t)-\dot{u}_{2}(a, t)\right]=0
\end{aligned}
$$

where $d$ is the damping coefficient of each identical dampers. The primes and dots in the above equations refer to partial derivatives with respect to the position coordinate $x$ and time $t$,respectively.

Assuming the axial displacements $u_{1}(x, t), u_{2}(x, t)$ and $u_{3}(x, t)$ are separable in space and time in the form of

$$
u_{j}(x, t)=U_{j}(x) e^{\lambda t} ; \quad j=1,2,3
$$

in which $U_{j}(x)$ and $\lambda$ correspond to unknown amplitude function and characteristic value respectively. Equation (1) takes the form of the following eigenvalue problem

$$
\begin{gathered}
d^{2} U_{j}(x) \\
d x^{2}
\end{gathered}-\beta^{2} U_{j}(x)=0 ; \quad j=1,2,3
$$

where

$$
\beta^{2}=\begin{gathered}
m \lambda^{2} \\
E A
\end{gathered}
$$

These differential equations have the general solutions

$$
\begin{aligned}
& U_{1}(x)=C_{1} e^{\beta x}+C_{2} e^{-\beta x} \\
& U_{2}(x)=C_{3} e^{\beta x}+C_{4} e^{-\beta x} \\
& U_{3}(x)=C_{5} e^{\beta x}+C_{6} e^{-\beta x}
\end{aligned}
$$

where $C_{1} \sim C_{6}$ are the integration constants to be determined by the boundary and matching conditions given in Eqs (2) to (9). These boundary and matching conditions in connection with the assumed solutions in Eq. (10) lead to the six equations in six unknowns $C_{1} \sim C_{6}$. Writing these as a matrix equation and equating the determinant to zero result in the characteristic equations for locally reacting damping system (Case 1)

$$
\begin{aligned}
& \sinh \bar{\beta}+\sinh (1-2 \bar{b}) \bar{\beta}-\sinh (1-2 \bar{a}) \bar{\beta}-\sinh (1+2 \bar{a}-2 \bar{b}) \bar{\beta} \\
& \quad+\frac{1}{D}[4 \cosh \bar{\beta}-2 \cosh (1-2 \bar{a}) \bar{\beta}-2 \cosh (1-2 \bar{b}) \bar{\beta}]+{ }_{D^{2}}^{1} \sinh \bar{\beta}=0
\end{aligned}
$$

and for non-locally reacting system (Case 2)

$$
2[\cosh \bar{\beta}+\cosh (1-\bar{a}-\bar{b}) \bar{\beta}-\cosh (1+\bar{a}-\bar{b}) \bar{\beta}]-\cosh (1-2 \bar{a}) \bar{\beta}-\cosh (1-2 \bar{b}) \bar{\beta}+\frac{2}{D} \sinh \bar{\beta}=0(17)
$$

where the notations

$$
\bar{\beta}=\beta L, \bar{a}={ }_{L}^{a}, \bar{b}=\stackrel{b}{L}, D=\underset{E A \beta}{d \lambda}=\underset{\sqrt{ } E A m}{d}
$$

are introduced for simplicity and $D$ can be considered as the dimensionless damping coefficient. 


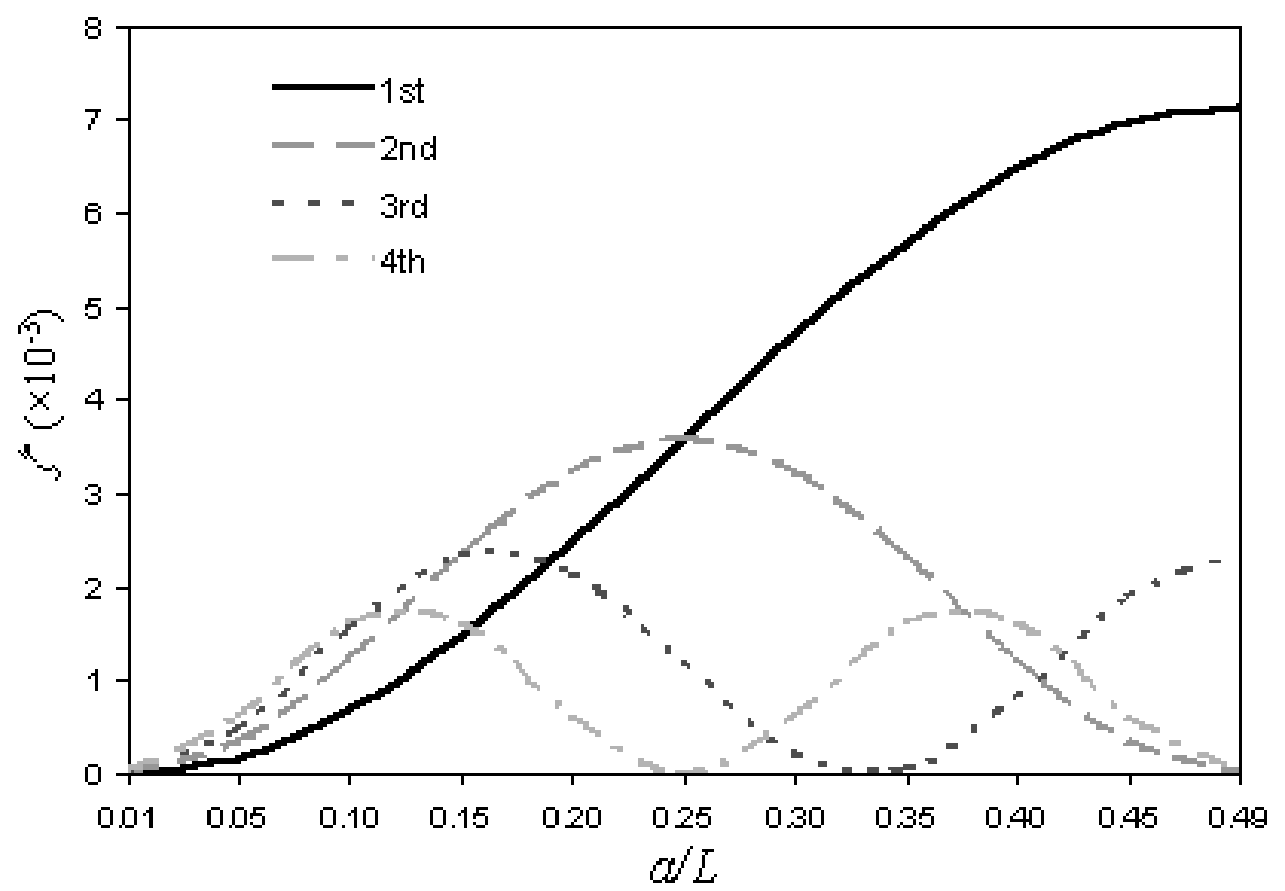

Fig. 2. Damping ratios versus damper locations for the first four eigenfrequencies of locally reacting damping.

In general, damped systems have complex eigenfrequencies and complex modes. In order to evaluate the complex frequencies of the system, complex frequency term $\bar{\beta}$ is considered as a complex number in the following form:

$$
\bar{\beta}=x+i y
$$

where $x$ and $y$ denotes the real and imaginary parts of the complex number respectively.

Now, Eq. (19) is inserted into the characteristic Eqs (16) and (17). Separating the real and imaginary parts of the equations, a pair of equations is obtained in terms of $x$ and $y$ for each Eqs (16) and (17). For the locally reacting damping system the following equation pair is obtained

$$
\begin{aligned}
& F(x, y)+F(x-2 \bar{b} x, y-2 \bar{b} y)-F(x-2 \bar{a} x, y-2 \bar{a} y)-F(x+2 \bar{a} x-2 \bar{b} x, y+2 \bar{a} y-2 \bar{b} y) \\
& \quad+\frac{1}{D}[4 G(x, y)-2 G(x-2 \bar{a} x, y-2 \bar{a} y)-2 G(x-2 \bar{b} x, y-2 \bar{b} y)]+{ }_{D^{2}}^{4} F(x, y)=0 \\
& H(x, y)+H(x-2 \bar{b} x, y-2 \bar{b} y)-H(x-2 \bar{a} x, y-2 \bar{a} y)-H(x+2 \bar{a} x-2 \bar{b} x, y+2 \bar{a} y-2 \bar{b} y) \\
& \quad+\frac{1}{D}[4 K(x, y)-2 K(x-2 \bar{a} x, y-2 \bar{a} y)-2 K(x-2 \bar{b} x, y-2 \bar{b} y)]+{ }_{D^{2}}^{4} H(x, y)=0
\end{aligned}
$$

where

$$
\begin{aligned}
& F(x, y)=\sinh (x) \cdot \cos (y) \\
& G(x, y)=\cosh (x) \cdot \cos (y) \\
& H(x, y)=\cosh (x) \cdot \sin (y) \\
& K(x, y)=\sinh (x) \cdot \sin (y)
\end{aligned}
$$

For the non-locally reacting damping system, the following equation pair is obtained: 


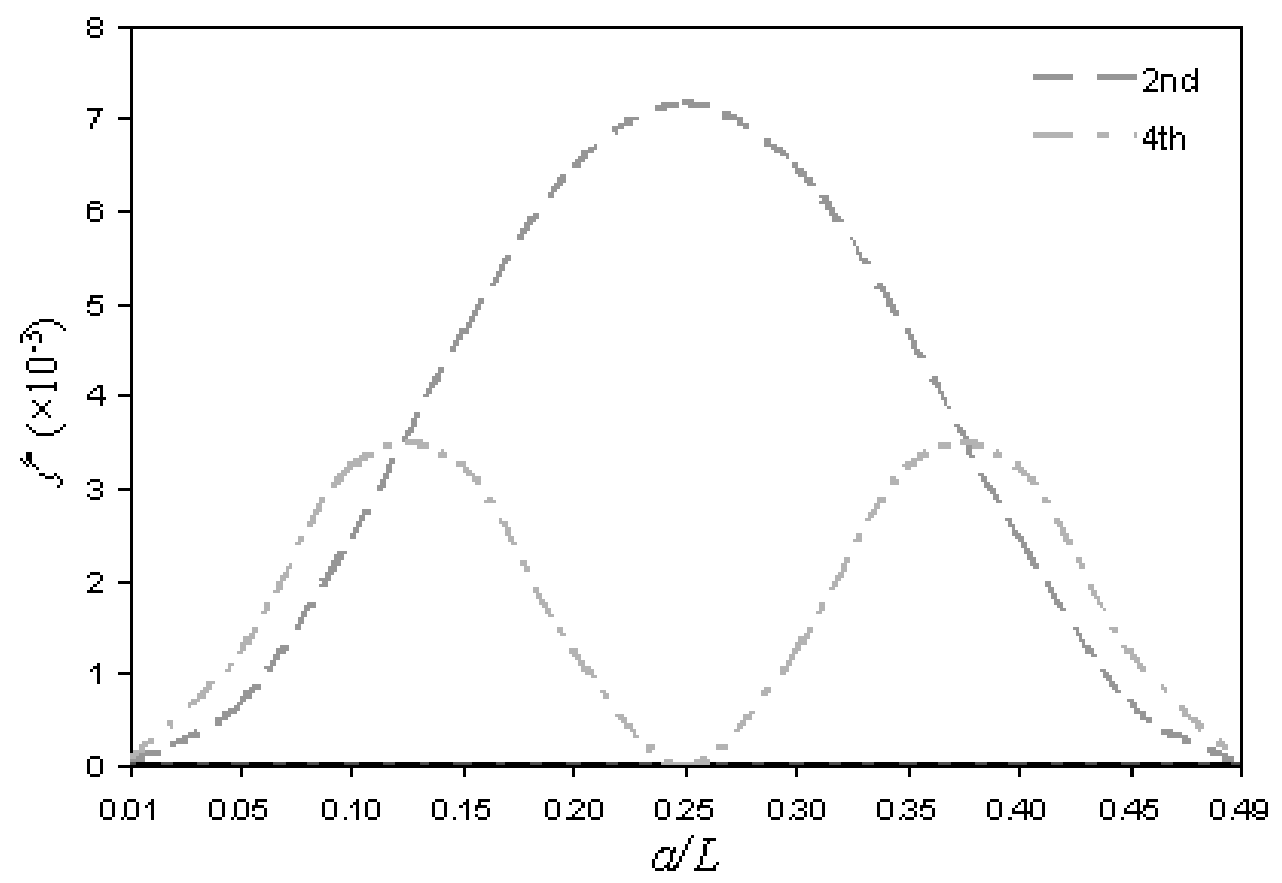

Fig. 3. Damping ratios versus damper locations for the first four eigenfrequencies of non-locally reacting damping.

$$
\begin{aligned}
& 2 G(x, y)+2 G(x-\bar{a} x-\bar{b} x, y-\bar{a} y-\bar{b} y)-2 G(x+\bar{a} x-\bar{b} x, y+\bar{a} y-\bar{b} y)-G(x-2 \bar{a} x, y-2 \bar{a} y) \\
& -G(x-2 \bar{b} x, y-2 \bar{b} y)+\frac{2}{D} F(x, y)=0 \\
& 2 K(x, y)+2 K(x-\bar{a} x-\bar{b} x, y-\bar{a} y-\bar{b} y)-2 K(x+\bar{a} x-\bar{b} x, y+\bar{a} y-\bar{b} y)-K(x-2 \bar{a} x, y-2 \bar{a} y) \\
& -K(x-2 \bar{b} x, y-2 \bar{b} y)+{ }_{D}^{2} H(x, y)=0
\end{aligned}
$$

Since the above equations are very complicated and in order to have a symmetrical system and solve the problem easier and faster, we assume that the dampers are located at the same distances from the ends. In this case,

$$
b=L-a \quad \text { or } \quad \bar{b}=1-\bar{a}
$$

Using this relation, Eqs (20) and (21) and Eqs (26) and (27) respectively take the following forms:

$$
\begin{aligned}
& F(x, y)-2 F(x-2 \bar{a} x, y-2 \bar{a} y)+F(x-4 \bar{a} x, y-4 \bar{a} y)+\frac{1}{D}[4 G(x, y)-4 G(x-2 \bar{a} x, y-2 \bar{a} y)] \\
& \quad+{ }_{D^{2}}^{4} F(x, y)=0 \\
& H(x, y)-2 H(x-2 \bar{a} x, y-2 \bar{a} y)+H(x-4 \bar{a} x, y-4 \bar{a} y)+\frac{1}{D}[4 K(x, y)-4 K(x-2 \bar{a} x, y-2 \bar{a} y)] \\
& \quad+{ }_{D^{2}}^{4} H(x, y)=0 \\
& G(x, y)-G(2 \bar{a} x, 2 \bar{a} y)-G(x-2 \bar{a} x, y-2 \bar{a} y)+{ }_{D}^{1} F(x, y)+1=0 \\
& K(x, y)-K(2 \bar{a} x, 2 \bar{a} y)-K(x-2 \bar{a} x, y-2 \bar{a} y)+{ }_{D}^{1} H(x, y)=0
\end{aligned}
$$

Each of the pairs Eqs (29) and (30) and Eqs. (31) and (32) constitutes a system of equations to be solved simultaneously. 

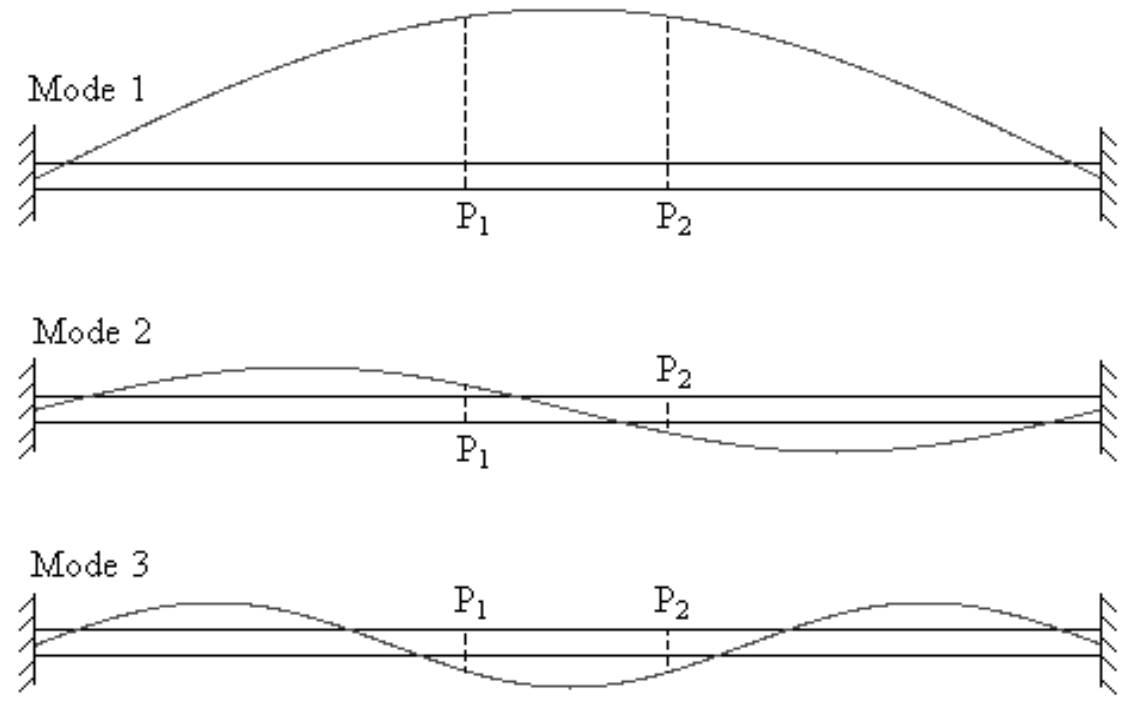

Mode 4

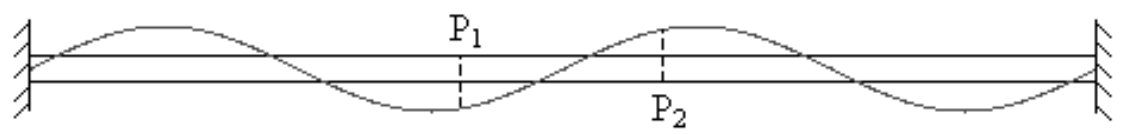

Fig. 4. First four mode shapes of the longitudinally vibrating undamped elastic rod.

\section{Optimal system parameters and numerical examples}

In this section, the numerical solutions of the equation pairs Eqs (29) and (30) for the locally reacting damping system and Eqs (31) and (32) for the non-locally reacting damping system are presented. For the numerical analysis, all system parameters for the rod are fixed. The parameters of the beam are taken from $[17,18]$ and are as follows. The beam length $L=50 \mathrm{~m}$, modulus of elasticity $E=2.1 \times 10^{8} \mathrm{~N} / \mathrm{m}^{2}$, cross sectional area $A=2.6 \mathrm{~m}^{2}$, and density $\rho=7850 \mathrm{~kg} / \mathrm{m}^{3}$. The damping coefficient is set to be $d=250 \mathrm{kNs} / \mathrm{m}$.

Using the numerical solutions with the help of Mathcad, the equation pairs Eqs (29) and (30) and Eqs (31) and (32) are solved simultaneously and the real $(x)$ and imaginary $(y)$ parts of complex eigenfrequencies $(\bar{\beta})$ are determined for the locally (Case 1) and non-locally (Case 2) damped elastic rod. The results of the numerical solutions are given in Table 1. The first four eigenfrequencies of damped elastic rod for both damping cases are presented in this table in the form of $x+i y$ for the selected dimensionless damper locations $a / L$ varying from 0.01 to 0.49 .

The complex eigenfrequency $\lambda$ of a vibrating system can be represented by the following well known form with the real and imaginary parts [15]

$$
\lambda=-\zeta \omega_{n} \pm i \sqrt{ } 1-\zeta^{2} \omega_{n}
$$

where $i=\sqrt{ }-1, \omega_{n}$ denotes the undamped natural frequency, and $\zeta$ denotes the damping ratio that can be evaluated by using the real and imaginary parts of the characteristic value as

$$
\zeta=\frac{|\operatorname{Re}(\lambda)|}{\sqrt{ } \operatorname{Re}(\lambda)^{2}+\operatorname{Im}(\lambda)^{2}}
$$

Applying Eq. (34), damping ratios are calculated from the eigenfrequencies listed in Table 1 for both locally and non-locally reacting damping cases. Figures 2 and 3 present the variation of damping ratios with respect to the selected damper locations for the first four eigenfrequencies of locally and non-locally reacting damping, respectively. 
Table 1

First four dimensionless eigenfrequencies of the locally (Case 1) and non-locally (Case 2) damped elastic rod

$\bar{\beta}_{1}$

$\begin{array}{cc}\mathrm{a} / \mathrm{L} & \text { Case } 1 \\ 0.01 & -0.000022+3.141593 \mathrm{i} \\ 0.05 & -0.000550+3.141593 \mathrm{i} \\ 0.10 & -0.002149+3.141597 \mathrm{i} \\ 0.15 & -0.004639+3.141601 \mathrm{i} \\ 0.20 & -0.007776+3.141602 \mathrm{i} \\ 0.25 & -0.011254+3.141593 \mathrm{i} \\ 0.30 & -0.014732+3.141577 \mathrm{i} \\ 0.35 & -0.017869+3.141560 \mathrm{i} \\ 0.40 & -0.020359+3.141552 \mathrm{i} \\ 0.45 & -0.021958+3.141562 \mathrm{i} \\ 0.49 & -0.022487+3.141585 \mathrm{i}\end{array}$

$\mathrm{a} / \mathrm{L}$

0.01

0.05

0.10

0.15

0.20

0.25

0.30

0.35

0.40

0.45

0.49

Case 1

$-0.000199+9.424778 \mathrm{i}$

$-0.004639+9.424795 \mathrm{i}$

$-0.014732+9.424825 \mathrm{i}$

$-0.021958+9.424793 \mathrm{i}$

$-0.020359+9.424764 \mathrm{i}$

$-0.011254+9.424778$

$-0.000550+9.424778 \mathrm{i}$

$-0.002149+9.424779 i$

$-0.007776+9.424753 \mathrm{i}$

$-0.017869+9.424713$

$-0.022309+9.424755 \mathrm{i}$
$0.000000+3.141593 i$

$0.000000+3.141593$

$0.000000+3.141593 i$

$0.000000+3.141593 i$

$0.000000+3.141593 i$

$0.000000+3.141593 i$

$0.000000+3.141593 i$

$0.000000+3.141593 \mathrm{i}$

$0.000000+3.141593$

$0.000000+3.141593 i$

$0.000000+3.141593$

$\bar{\beta}_{3}$
Case 2

$0.000000+9.424778 \mathrm{i}$

$0.000000+9.424778 \mathrm{i}$

$0.000000+9.424778 \mathrm{i}$

$0.000000+9.424778 \mathrm{i}$

$0.000000+9.424778 \mathrm{i}$

$0.000000+9.424778 \mathrm{i}$

$0.000000+9.424778 \mathrm{i}$

$0.000000+9.424778$

$0.000000+9.424778 \mathrm{i}$

$0.000000+9.424778 \mathrm{i}$

$0.000000+9.424778 \mathrm{i}$ $\bar{\beta}_{2}$

Case 1

Case 2

$-0.000089+6.283185 \mathrm{i}$

$-0.002149+6.283191 \mathrm{i}$

$-0.007776+6.283210 \mathrm{i}$

$-0.014732+6.283217 \mathrm{i}$

$-0.020359+6.283199 \mathrm{i}$

$-0.022508+6.283185 \mathrm{i}$

$-0.020359+6.283199 \mathrm{i}$

$-0.014732+6.283217 \mathrm{i}$

$-0.007776+6.283210 \mathrm{i}$

$-0.002149+6.283191 \mathrm{i}$

$-0.000089+6.283185 \mathrm{i}$

$-0.000177+6.283186 \mathrm{i}$

$-0.004299+6.283208 \mathrm{i}$

$-0.015553+6.283285 \mathrm{i}$

$-0.029464+6.283311 \mathrm{i}$

$-0.040719+6.283239 \mathrm{i}$

$-0.045018+6.283185 \mathrm{i}$

$-0.040719+6.283239 \mathrm{i}$

$-0.029464+6.283311 \mathrm{i}$

$-0.015553+6.283285 \mathrm{i}$

$-0.004299+6.283208 \mathrm{i}$

$-0.000177+6.283186 \mathrm{i}$

$\bar{\beta}_{4}$

Case 1

Case 2

$-0.000354+12.566371 \mathrm{i}$

$-0.007776+12.566404 \mathrm{i}$

$-0.020359+12.566412 \mathrm{i}$

$-0.020359+12.566344 \mathrm{i}$

$-0.007776+12.566362 \mathrm{i}$

$-0.000001+12.566371 \mathrm{i}$

$-0.020359+12.566344 \mathrm{i}$

$-0.007776+12.566362 \mathrm{i}$

$-0.020359+12.566411 \mathrm{i}$

$-0.007776+12.566404 \mathrm{i}$

$-0.000354+12.566371 \mathrm{i}$
$-0.000707+12.566373 \mathrm{i}$

$-0.015552+12.566504 \mathrm{i}$

$-0.040720+12.566532 \mathrm{i}$

$-0.040720+12.566263 \mathrm{i}$

$-0.015553+12.566337 \mathrm{i}$

$-0.000005+12.566371 \mathrm{i}$

$-0.040720+12.566263 \mathrm{i}$

$-0.015553+12.566337 \mathrm{i}$

$-0.040720+12.566532 \mathrm{i}$

$-0.015552+12.566504$

$-0.000707+12.566373 \mathrm{i}$

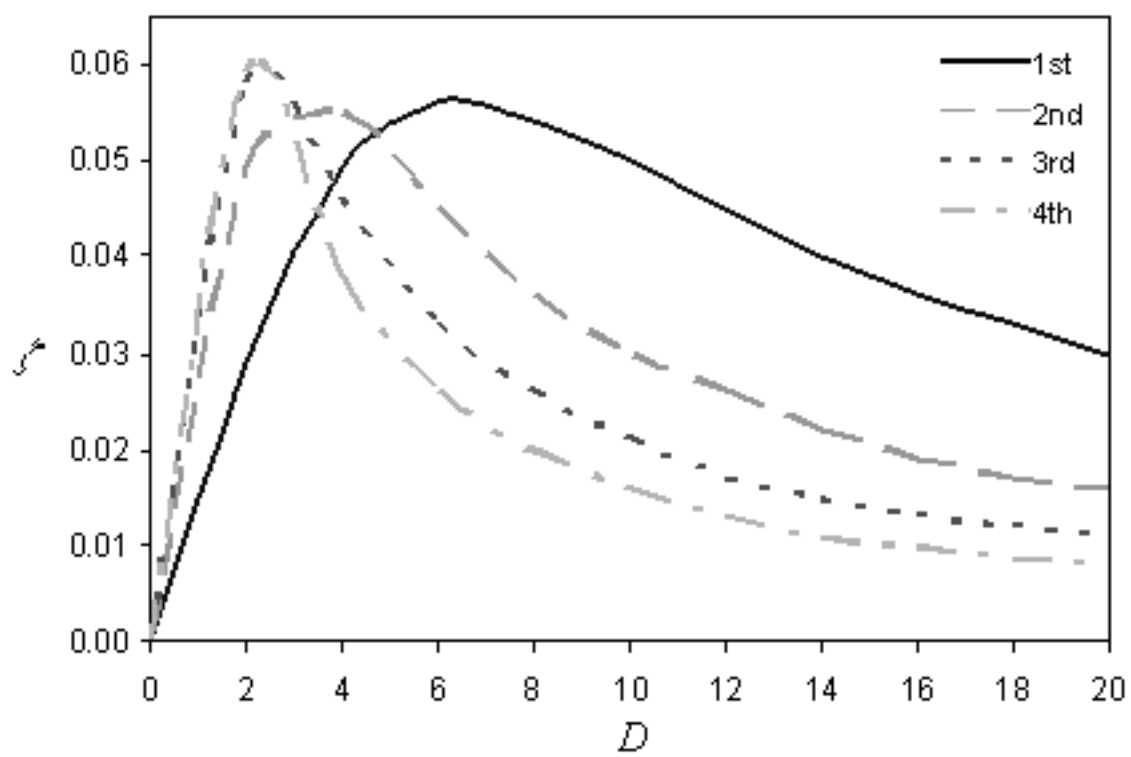

Fig. 5. Damping ratios versus dimensionless damping coefficient for the first four eigenfrequencies of locally reacting damping for $a=0.05$.

From Fig. 2, it can be seen that for the locally reacting damping and for the symmetrically located viscous dampers, the change of damping ratio with respect to the damper location for the first four eigenfrequencies are very similar to first four mode shapes of the undamped system, which are shown in Fig. 4. In fact, if the absolute values of the first four mode shapes are plotted for only the left half of the undamped rod, then exactly the same shape curves shown in Fig. 2 will be evaluated. As a result, it is clear that the damping ratio for ith mode is linearly proportional to $\left|U_{i}(a)\right|$. 
Table 2

Optimal damping ratio and damping coefficient values for the first four eigenfrequencies of the locally (Case 1) and non-locally (Case 2) damped systems

\begin{tabular}{cccll} 
& \multicolumn{2}{c}{ Case 1 } & \multicolumn{2}{c}{ Case 2 } \\
Eigenfrequency & $\left.D\right|_{\text {opt }}$ & $\left.\zeta\right|_{\text {opt }}$ & $\left.D\right|_{\text {opt }}$ & $\left.\zeta\right|_{o p t}$ \\
1st & 6.42 & 0.056 & 0.000 & 0.000 \\
2nd & 3.10 & 0.057 & 1.97 & 0.056 \\
3rd & 2.04 & 0.058 & 0.000 & 0.000 \\
4th & 1.50 & 0.062 & 1.71 & 0.040
\end{tabular}

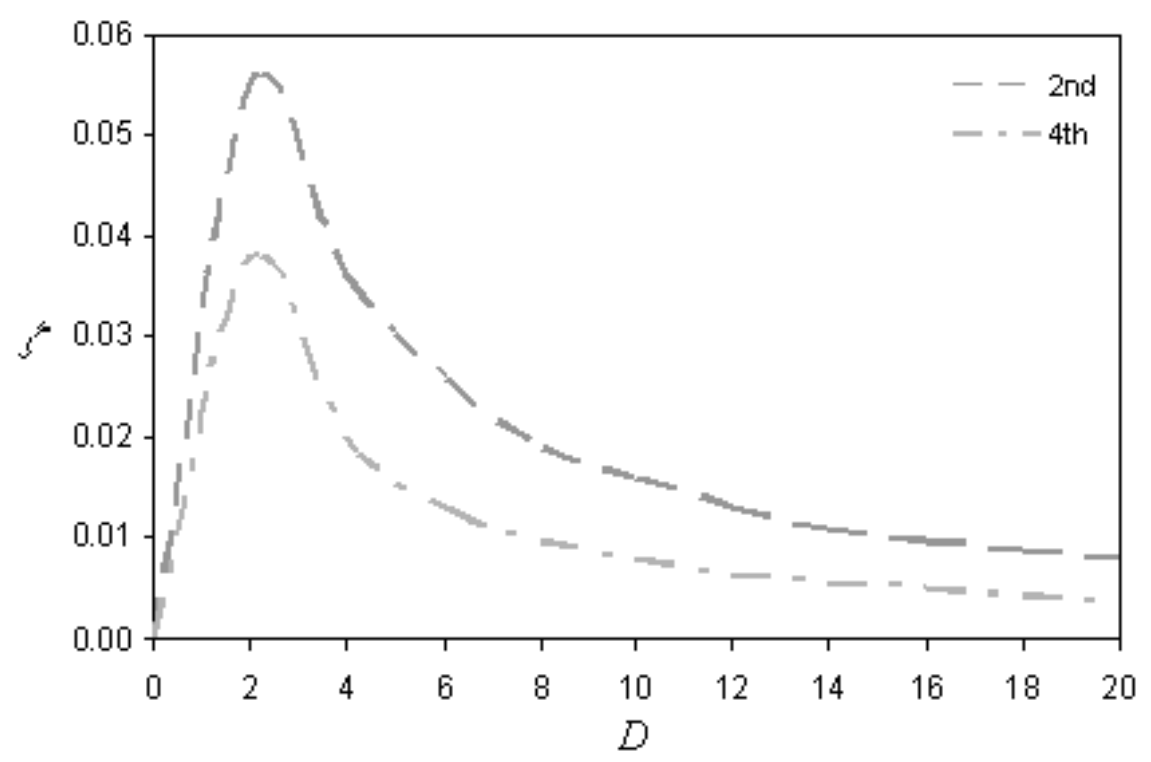

Fig. 6. Damping ratios versus dimensionless damping coefficient for the first four eigenfrequencies of non-locally reacting damping for $a=0.05$.

For the non-locally reacting damping case, Fig. 3 shows similar damping ratio versus damper location plots for the 2nd and 4th eigenfrequencies as in Fig. 2. However, for the 1st and 3rd eigenfrequencies of the non-locally damped case, the damping ratio approaches zero regardless of the damper location. Such a result is obvious since the real parts of the 1st and 3rd eigenfrequencies listed in Table 1 for Case 2 are all zero. This situation can be explained by referring to the previous statement given for the locally reacting damping system that there is a direct and linear correlation with the damping ratio and mode shape of the undamped system. The dampers are located at symmetrical points from the ends of the rod and it can be seen clearly from Fig. 4 that, the symmetrical points from the ends of the rod always move in the same direction and in the same magnitude for the undamped case. The symmetrically located dampers have no damping effect on the system for 1st and 3rd eigenfrequencies of the non-locally damped system. The result for these cases are consistent with the results of Gürgöze [16] in which it is stated that such a non-locally damped system can not be asymptotically stable.

Figures 5 and 6 illustrate damping ratios with respect to dimensionless damping coefficient for the first four eigenfrequencies of locally and non-locally damped systems, respectively. For fixed and symmetrical damper locations with $a / L=0.05$, damping ratios are calculated for varying D from 0.001 to 20 .

It can be seen from Fig. 5 that there are optimal values for the damping coefficient for each eigenfrequency and consequently, increasing the damping coefficient does not result in better damping ratios. The optimal damping ratio and damping coefficient values for the first four eigenfrequencies of the locally damped system are given in Table 2. The optimal damping coefficient for the first eigenfrequency is very close to that found by Y üksel and Gürgöze [13].

Figure 6 presents similar results for the non-locally damped system with locally damped system. Damping ratio can not be improved always with the increasing damping coefficient and there are optimal values for the damping 
coefficient for each eigenfrequency. For the 1st and 3rd eigenfrequencies of the non-locally damped case, the damping ratio goes to zero regardless of damping coefficient as explained above. The optimal damping ratio and damping coefficient values for the 2 nd and 4 th eigenfrequencies are also given in Table 2.

\section{Conclusions}

In this study, eigencharacteristics of longitudinally vibrating rods are analyzed for two alternative damping cases, namely, locally and non-locally damping. Considering the rod as a continuous system, the complex eigenfrequencies are determined as the solution of a characteristic equation for the locally and non-locally damping cases. The resulting eigenfrequencies are presented in Table 1. The variations of the damping ratios with respect to damper locations for the first four eigenfrequencies are plotted.

It is concluded that there is a direct correlation between the damping ratio and mode shape of the undamped system for the longitudinally vibrating elastic rod for both locally and non-locally damping cases. Determination of the correlation between the damping ratio and mode shape for the systems considered needs further study.

In the beginning of this study, the non-locally damped system is considered to be an alternative to the locally damped system from the point of view of design flexibility and installation easiness. However, it is seen that, the nonlocally damped system can not be asymptotically stable for the $1 \mathrm{st}$, 3rd and remaining odd numbered eigenfrequencies regardless of the damper location and damping coefficient, if the dampers are located symmetrically. Therefore, for the non-locally damping case, the dampers should not be located symmetrically from the ends of the rods.

The variations of the damping ratios with respect to damping coefficients for the first four eigenfrequencies are plotted. The optimal values for external damping coefficients of viscous dampers are determined for a given damper location for both locally and non-locally damping systems and results are presented in a table.

\section{References}

[1] J.H. Zarek and B.M. Gibbs, The derivation of eigenvalues and mode shapes for the bending motion of a damped beam with general end conditions, Journal of Sound and Vibration 78 (1981), 185-196.

[2] G. Oliveto, A. Satini and E. Tripodi, Complex modal analysis of a flexural vibrating beam with viscous end conditions, Journal of Sound and Vibration 200(3) (1997), 327-345.

[3] B. Yang and X. Wu, Transient response of one dimensional distributed systems: A closed form eigenfunction expansion realization, Journal of Sound and Vibration 208(5) (1997), 763-776.

[4] I. Kovacs, Zur Frage der Seilschwigungen und der Seildampfung, Die Bautechnik 59(10) (1982), $325-332$.

[5] B.N. Pacheco, Y. Fujino and A. Sulekh, Estimation curve for modal damping in stay cables with viscous damper, Journal of Structural Engineering 119(6) (1993), 1961-1979.

[6] S. Krenk, Vibrations of a taut cable with an external damper, Journal of Applied Mechanics 67 (2000), $772-776$.

[7] M.J. Casarella and P.A. Laura, Drag on an oscillating rod with longitudinal and torsional motion, Journal of Hydronautics 3(4) (1969), $180-183$.

[8] J.E. Goeller and P.A. Laura, Dynamic stress and displacements in a two-material cable system subjected to longitudinal excitation, The Journal of the Acoustical Society of America 46(2) (1969), 284-292.

[9] R. Singh, W.M. Lyons and G. Preater, Complex eigensolution for longitudinally vibrating bars with a viscously damped boundary, Journal of Sound and Vibration 133 (1989), 364-367.

[10] A.J. Hull, A closed form solution of a longitudinal bar with a viscous boundary condition, Journal of Sound and Vibration 169(1) (1994), 19-28.

[11] N.A. Hzzal and M. Gürgöze, Lumped parameter representation of a longitudinally vibrating elastic rod viscously damped in-span, Journal of Sound and Vibration 216(2) (1998), 328-336.

[12] S. Adhikari and J. Woodhouse, Identification of Damping: Part 1, Viscous Damping. Journal of Sound and Vibration 243(1) (2001), 43-61.

[13] Ş. Yüksel and M. Gürgöze, Continuous and discrete models for longitudinally vibrating elastic rods viscously damped in-span, Journal of Sound and Vibration 257(5) (2002), 996-1006.

[14] R.E.D. Bishop and D.C. Johnson, The Mechanics of Vibration, Cambridge University Press, 1960.

[15] D.J. Inman, Engineering Vibration, Prentice Hall, Second Edition, 2001.

[16] M. Gürgöze, Letters to editor- "Another solution to the finite residual motion of A damped two degree of freedom vibrating system", Journal of Sound and Vibration 169(3) (1994), 409-410.

[17] F. Sadek and B. Mohraz, Semiactive control algorithms for structures with variable dampers, Journal of Engineering Mechanics 124(9) (1998), 981-990.

[18] S. Kojima, Dynamic Analysis of the Bridge with Frictional Support, Research Report, Chuo University Engineering, Tokyo, Japan, 2000. 

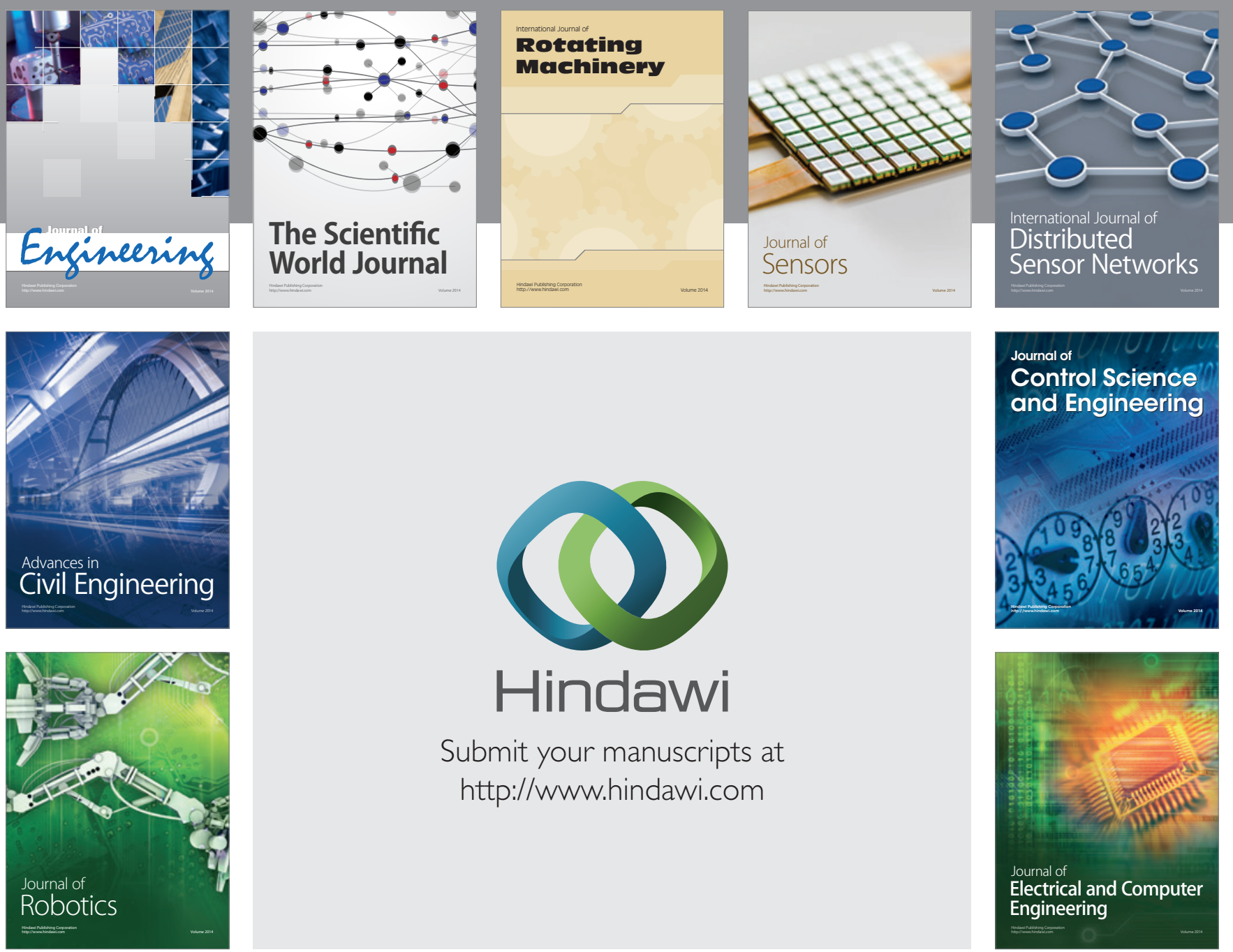

Submit your manuscripts at

http://www.hindawi.com
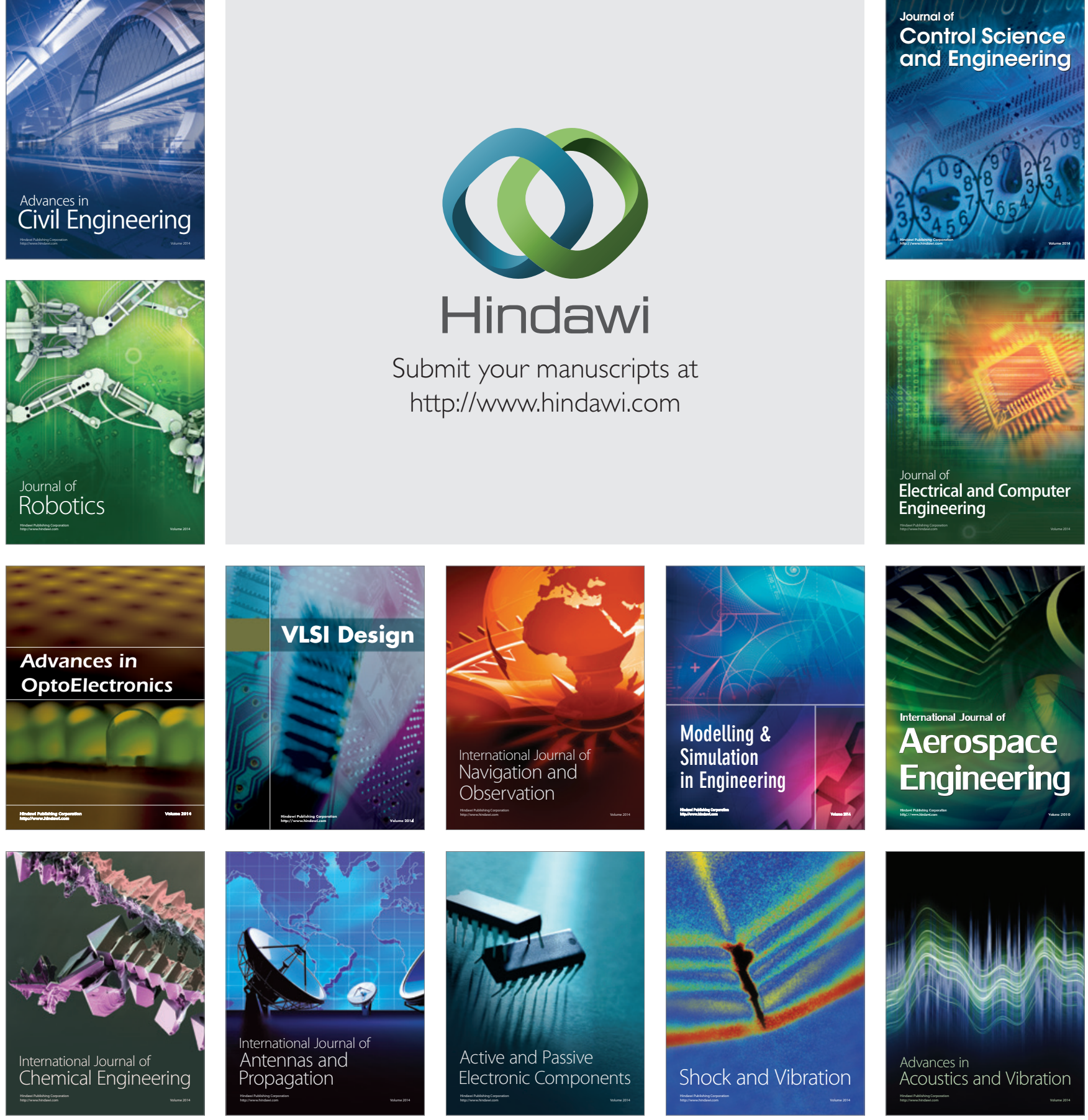